\title{
Posterior Reversible Encephalopathy Syndrome in a Patient with Newly Diagnosed HIV Infection and End Stage Renal Disease
}

\author{
Mohankumar Kurukumbi, ${ }^{1}$ Maria I. Castellanos, ${ }^{1}$ Amanda K. Crawford, ${ }^{1}$ \\ Shreyas D. Gowdar, ${ }^{2}$ and Annapurni Jayam-Trouth ${ }^{1}$ \\ ${ }^{1}$ Department of Neurology, Howard University Hospital, 2041 Georgia Avenue Northwest, Washington, DC 20001, USA \\ ${ }^{2}$ Department of Internal Medicine, Howard University Howard, 2041 Georgia Avenue Northwest, Washington, DC 20001, USA
}

Correspondence should be addressed to Mohankumar Kurukumbi; mohan311@gmail.com

Received 8 March 2013; Accepted 8 April 2013

Academic Editors: J. E. Cohen and M. Moonis

Copyright (C) 2013 Mohankumar Kurukumbi et al. This is an open access article distributed under the Creative Commons Attribution License, which permits unrestricted use, distribution, and reproduction in any medium, provided the original work is properly cited.

\begin{abstract}
Posterior reversible encephalopathy syndrome (PRES) is a clinicoradiological syndrome in which patients present with an acute or subacute clinical presentation of seizures, visual disturbances, headache, and altered mental status. The pathophysiology of PRES may be explained by endothelial dysfunction that leads to transudation of fluids and protein, resulting in vasogenic cerebral edema. PRES is typically associated with many conditions such as hypertension, uremia, immunosuppressive drugs, and sepsis. This is a case report of a 39-year-old woman with untreated HIV infection and end-stage renal disease (ESRD) who developed PRES with a normal blood pressure and no other known causes of PRES. Untreated HIV is associated with known endothelial dysfunction and we believe that this, in combination with her untreated end-stage renal disease, contributed to her unique presentation of PRES. Although uncommon in HIV-infected patients and challenging to diagnose, prompt recognition of PRES is critical to provide appropriate care and ensure reversibility of the vasogenic edema seen in PRES.
\end{abstract}

\section{Introduction}

Posterior reversible encephalopathy syndrome (PRES) is a clinicoradiological syndrome originally described in 1996 by Hinchey et al. [1]. Patients with PRES have an acute or subacute clinical presentation of altered mental status, encephalopathy, headache, new onset seizures, and visual disturbances in association with neuroimaging studies consistent with white matter cerebral edema [1].

The hallmark of this rare clinical entity is the reversibility once precipitating factors have been controlled.

There have been few case reports in which PRES has developed in HIV-infected patients [2-5], including one case in which HIV itself was a risk factor for the development of the syndrome [6]. Here we report a case of PRES in a patient whose only risk factor for development of PRES is HIV and renal failure.

\section{Case Presentation}

A thirty-nine-year-old African American female with no significant past medical history presented to the emergency department cachectic and "feeling sick" for two weeks with generalized fatigue, malaise, and productive cough with hemoptysis. Review of systems was positive for anorexia, unquantified weight loss, nausea, vomiting, and dizziness. She had no history of hypertension, hematologic disorders, or cancers in herself or in her family.

The clinical examination at the time of admission revealed severe pallor and oral candidiasis with the rest of the examination benign. Her mental status was normal and she was alert and oriented to person, place, and time. Medical research scale (MRC) grade was $4 / 5$ in all extremities, likely secondary to deconditioning and severe anemia. The patient's vital signs were normal, including a blood pressure of 122/65 mm Hg. 
On admission, laboratory analysis revealed severe abnormalities including blood urea nitrogen (BUN) of $138 \mathrm{mmol} / \mathrm{L}$, serum creatinine of $22.3 \mu \mathrm{mol} / \mathrm{L}$, hemoglobin of $4.9 \mathrm{~g} / \mathrm{dL}$, and hematocrit of $16.4 \%$ with a normocytic anemia. Arterial blood gas revealed $\mathrm{pH}$ 7.17, $\mathrm{pCO}_{2} 18.8 \mathrm{~mm} \mathrm{Hg}, \mathrm{pO}_{2}$ $143 \mathrm{~mm} \mathrm{Hg}$, and oxygen saturation of 99 percent on room air. The other laboratory parameters including white blood count, chemistry panel, platelets, and liver function tests were within normal limits. Urine toxicology screen and pregnancy tests were negative.

She was admitted to the intensive care unit and placed on droplet precautions until tuberculosis was ruled out. She was given 4 units of packed red blood cells after ruling out reversible causes of blood loss. Gynecologic history included eleven live births with poor antenatal care. During the hospital stay, the patient was diagnosed, for the first time, with new onset ESRD classified as Stage 5 chronic kidney disease. It had gone unchecked and the patient was unaware of any kidney issues. During this admission, She received multiple rounds of dialysis which brought her blood urea nitrogen and creatinine ratio (BUN/Cr) to near normal ranges over 3 days. Also, due to the patient's oral candidiasis her HIV status was checked. HIV ELISA and Western Blot were positive with a CD4 T lymphocyte count of 112 cells/ $\mu \mathrm{L}$ and a viral load of 780,000 cells $/ \mathrm{mm}^{3}$.

The patient was stable for the first 4 days until she had 2 seizures within 24 hours and became nonverbal, uncooperative, and encephalopathic. By this point, her BUN/Cr was brought down to 25/6.2 from the rounds of dialysis. Her blood pressure during the period of seizure was $152 / 96 \mathrm{~mm} \mathrm{Hg}$, which was thought to be secondary to the seizures, and electrolytes were within normal limits. She responded to painful stimuli, pupils were equally reactive, deep tendon reflexes intact, MRC rated 3/5, and there was no papilledema or neck stiffness. Keppra was started for seizure control.

Computed tomography (CT) without contrast showed white matter hypodensity in the posterior parietal-occipital lobes with the remaining findings within normal limits (Figure 1). A CT performed the following day confirmed the presence of hypodense regions but with newly noted small hemorrhages within the parietal and occipital regions. Magnetic resonance imaging (MRI) without contrast revealed areas of $\mathrm{T} 2$ prolongation in the subcortical white matter, predominantly in the occipital and parietal regions with scattered foci of susceptibility artifact bilaterally in the occipital lobes. The largest focus measured $10 \mathrm{~cm} \times 0.6 \mathrm{~cm}$ in the left occipital lobe (Figure 2). A repeat MRI with gadolinium showed a stable image with no pathologic enhancement and the foci of hemorrhage remained stable (Figure 3 ).

Given that PRES is a diagnosis of exclusion and based on clinical and radiographic findings, infectious causes of this acute change in mental status were ruled out. Cerebrospinal fluid analysis showed protein of $164 \mathrm{mg} / \mathrm{dL}$, glucose of $49 \mathrm{mg} / \mathrm{dL}, 630 \mathrm{RBCs} / \mathrm{mm}^{3}, 0 \mathrm{WBCs} / \mathrm{mm}^{3}$, and $\mathrm{LDH}$ of 37 U/L. No acid fast bacilli were seen. Polyomavirus, herpes simplex virus 1 and 2, cytomegalovirus, Epstein-Barr virus, Toxoplasma Gondii IgG, and VDRL were negative. Two sets of

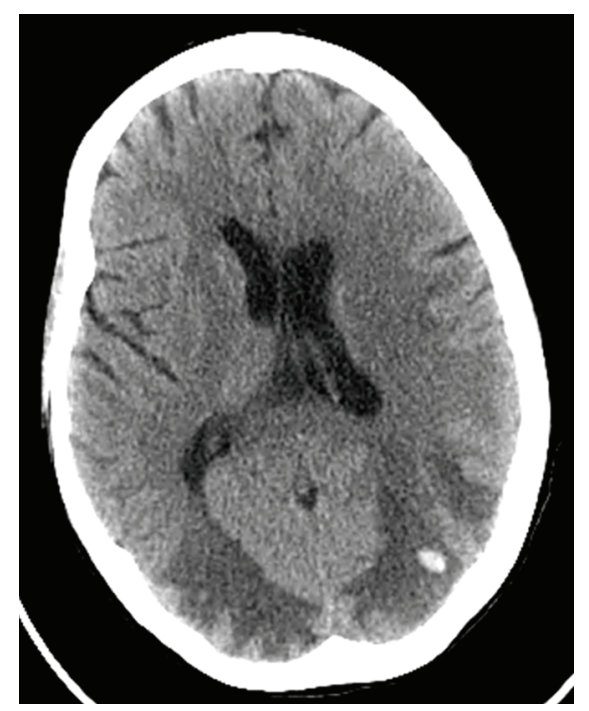

FIGURE 1: CT without contrast, hypodensity noted in the posterior parietal-occipital regions bilaterally and a small hemorrhage in the left parietooccipital region.

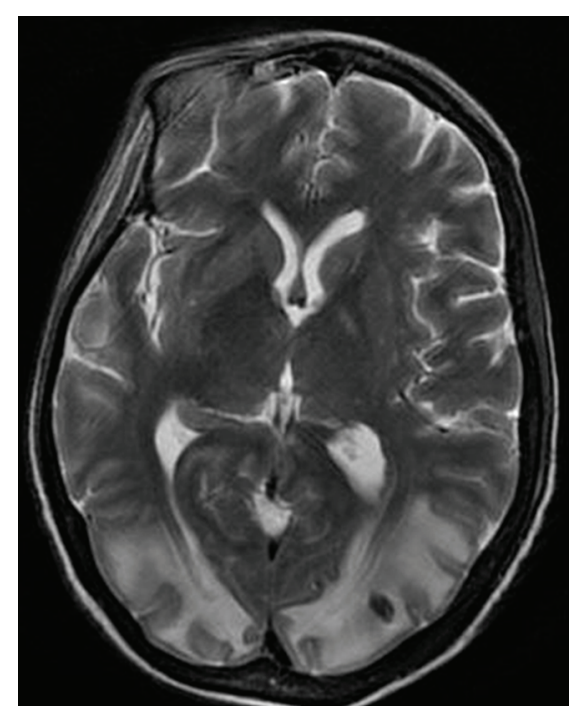

FIGURE 2: Axial T2-weighted MRI image without contrast demonstrating hyperintensity in the occipital and parietal regions bilaterally, with small foci hemorrhage in the parieto-occipital region.

blood and urine cultures were negative. There were no further complications with mental status and her cognitive function recovered completely. The patient's vital signs, hemoglobin, and BUN/Cr were stable when she was discharged to home with follow up at the HIV and renal clinics.

\section{Discussion}

Patients with PRES have an acute or subacute clinical presentation of altered mental status, encephalopathy, headache, new onset seizures, and visual disturbances in association with neuroimaging studies consistent with white matter cerebral edema [1]. Findings in MRI imaging reveal hyperintense 


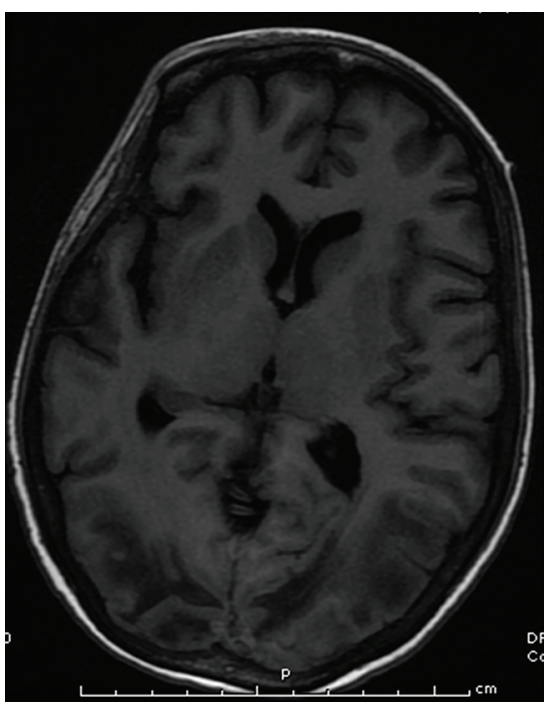

(a)

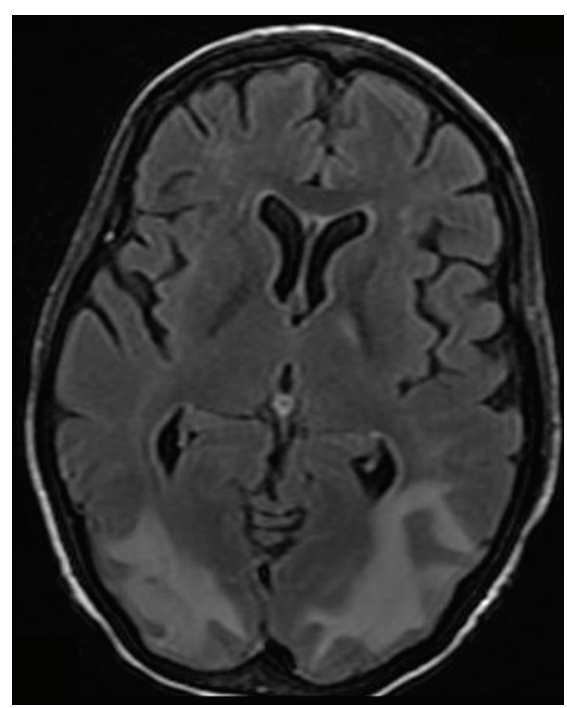

(b)

FIgURE 3: (a) Axial T1-weighted MRI image showing hypointensity in the occipital and parietal regions bilaterally. (b) Axial FLAIR with gadolinium MRI image showing lack of enhancement.

signaling on T2-weighted and fluid-attenuated inversion recovery (FLAIR) images and hypointense signaling in T1weighted images, indicative of cerebral edema. The regions most commonly affected are the parietal and occipital lobes bilaterally; although, the frontal lobe, temporal lobe, cerebellum, and brainstem regions have also been reported to be affected [7].

Many conditions exist that are commonly associated with PRES, including hypertensive encephalopathy, preeclampsia/eclampsia, immunosuppressive drugs, renal disease, fluid overload, autoimmune diseases, and sepsis [8]. The clinical and neuroimaging findings tend to be reversible once the underlying cause of PRES is identified and treated. Reversibility of the clinical and radiological findings may take between 5 days and 17 months, although there have been reports where normal function is not fully regained [9].

The exact pathophysiology of PRES remains unknown. However, three hypotheses exist that may explain the radiological findings in PRES and are related to (1) disorganized cerebrovascular autoregulation, (2) endothelial dysfunction, and (3) vasospasm related to acute increases in blood pressure $[1,6,10]$. Sudden elevation of systemic blood pressure causes cerebrovascular autoregulation failure and breakdown of the blood brain barrier, resulting in hyperperfusion. This loss of autoregulation more prominently affects the parieto-occipital regions because there is decreased sympathetic innervation in the posterior cerebral arterial circulation [11]. The loss of cerebral autoregulation leads to arteriolar vasodilation and endothelial dysfunction, resulting in transudation of fluids and proteins, causing cerebral vasogenic edema [11]. Moreover, cerebral vasospasm can lead to hypoxia and cytotoxic ischemia leading to edema predominantly found in the parietal-occipital regions and watershed areas of the brain [10].

In the setting of renal failure, azotemia may cause interstitial brain edema by increasing the permeability of capillaries and cytotoxic edema by direct injury of the brain parenchyma [12]. The overall occurrence of PRES is currently unknown [9]. However, a retrospective study of 36 patients with PRES reported $45 \%$ (17 patients) had the comorbidity of renal failure [7], making renal failure a common condition found in patients diagnosed with PRES. There is one case report of recurrent PRES in a patient with HIV on dialysis [13]. PRES can be a complication of dialysis-dependent patients, but it is due to uremia that dialysis-dependent patients develop as opposed to dialysis disequilibrium syndrome that occurs within hours of treatment [14].

There are several case reports that describe PRES occurring in patients infected with HIV [2-5]. Three case reports are in the setting of hypertensive crisis $[2,3]$, one of which was indinavir induced [3]. Other reports have been in association with hypercalcemia and disseminated coinfection with tuberculosis that developed PRES after being started on anti-TB and ART therapy $[4,5]$. However, none of these reports document HIV alone being a risk factor for PRES. Nightingale et al. reported a case in which HIV alone may constitute a risk factor for PRES [6].

HIV has been shown to be an independent risk factor for endothelial dysfunction and vascular disease [15]. HIV is known to induce endothelial dysfunction and apoptosis; HIV may infect endothelial cells and induce endothelial inflammation as noted by increased levels of circulating endothelial inflammatory markers [16]. Furthermore, bloodbrain barrier dysfunction is commonly seen in HIV-infected individuals and HIV is known to increase the permeability of the endothelium [15].

In our patient, who presented with renal failure due to HIV nephropathy, the development of altered sensorium and seizures occurred on day 4 of hospitalization. Of note, our patient did not have a severe elevation of blood pressure and her BUN/creatinine ratio was 25/6.2. PRES can be seen in the absence of hypertension in $20 \%-40 \%$ of cases of patients [9]. 
Our patient did not present with any other associated etiologies known to cause PRES. Therefore, it is within reason to infer that our patient's severely immunocompromised state and coexisting renal failure contributed significantly to endothelial dysfunction and resulted in the development of PRES.

Other rare contributing factors were considered in our patient. PRES has been observed as a very rare neurological complication of blood transfusions. In these case reports, the patients had a history of severe chronic anemia and were transfused with a high volume of blood in a short period of time [17]. The hypothesis that may explain this phenomenon is related to volume overload that results in the cerebral dysautoregulation due to a rapid increase in hemoglobin [17]. Our patient received 4 units of blood four days prior to the onset of seizures and altered sensorium. We believe that the blood transfusions she received did not contribute to the development of PRES as she received blood products over a gradual period of time.

\section{Conclusion}

The underlying mechanism of PRES, in our patient, could be due to endothelial dysfunction caused by uncontrolled, undiagnosed, and untreated HIV infection. Along with HIV, renal failure also contributed to endothelial dysfunction. To our knowledge, this is only the second case of PRES seen in an HIV-infected patient without concomitant hypertension. PRES should be considered in patients who are normotensive and HIV should be more widely recognized as a risk factor for the development of PRES. The presence of HIV makes the diagnosis of PRES challenging as other conditions may mimic similar clinicoradiological presentations and prompt recognition of this condition is critical to ensure reversibility of the vasogenic edema seen in PRES.

\section{Abbreviations}

PRES: Posterior reversible encephalopathy syndrome

HIV: Human immunodeficiency virus

ESRD: End-stage renal disease

CT: Computed tomography

MRI: Magnetic resonance imaging

BUN: Blood urea nitrogen

Cr: Creatinine

TB: Tuberculosis

ART: Antiretroviral therapy

FLAIR: Fluid-attenuated inversion recovery

ELISA: Enzyme linked immunosorbent assay

MRC: Medical research scale

IgG: Immunoglobulin G

LDH: Lactate dehydrogenase

VDRL: Venereal disease research laboratory

RBC: Red blood cell

WBC: White blood cell.

\section{Consent}

Written informed consent was obtained from the patient for publication of this case report and accompanying images.

\section{Disclosure}

The authors declare that they have no competing interests.

\section{Authors' Contribution}

M. Castellanos and A. Crawford, contributed extensive review of literature, paper drafting and share equal second authorship. M. Kurukumbi, primarily managed the patient and overviewed the paper. A. Jayam-Trouth, and S. Gowdar, overviewed the paper.

\section{References}

[1] J. Hinchey, C. Chaves, B. Appignani et al., "A reversible posterior leukoencephalopathy syndrome," The New England Journal of Medicine, vol. 34, no. 8, pp. 494-500, 1996.

[2] A. L. Ridolfo, F. Resta, L. Milazzo et al., "Reversible posterior leukoencephalopathy syndrome in $2 \mathrm{HIV}$-infected patients receiving antiretroviral therapy," Clinical Infectious Diseases, vol. 46, no. 2, pp. e19-e22, 2008.

[3] V. Giner, C. Fernández, M. J. Esteban et al., "Reversible posterior leukoencephalopathy secondary to indinavir-induced hypertensive crisis: a case report," American Journal of Hypertension, vol. 15, no. 5, pp. 465-467, 2002.

[4] M. Choudhary and F. Rose, "Posterior reversible encephalopathic syndrome due to severe hypercalcemia in AIDS," Scandinavian Journal of Infectious Diseases, vol. 37, no. 6-7, pp. 524526, 2005.

[5] S. Guerriero, L. Ciracì, T. Centoducati et al., "Bilateral visual loss as presenting symptom of posterior reversible encephalopathy syndrome in a patient with HIV/Tuberculosis coinfection: a case report," Case Reports in Ophthalmological Medicine, vol. 2012, Article ID 850176, 4 pages, 2012.

[6] S. Nightingale, C. Wood, and J. Ainsworth, "The posterior reversible encephalopathy syndrome in HIV infection," BMJ Case Reports, 2012.

[7] W. S. Bartynski and J. F. Boardman, "Distinct imaging patterns and lesion distribution in posterior reversible encephalopathy syndrome," American Journal of Neuroradiology, vol. 28, no. 7, pp. 1320-1327, 2007.

[8] V. H. Lee, E. F. M. Wijdicks, E. M. Manno, and A. A. Rabinstein, "Clinical spectrum of reversible posterior leukoencephalopathy syndrome," Archives of Neurology, vol. 65, no. 2, pp. 205-210, 2008.

[9] S. Legriel, F. Pico, and E. Azoulay, "Understanding posterior reversible encephalopathy syndrome," Annual Update in Intensive Care and Emergency Medicine, vol. 26, pp. 631-653, 2011.

[10] K. Naidu, J. Moodley, P. Corr, and M. Hoffmann, "Single photon emission and cerebral computerised tomographic scan and transcranial Doppler sonographic findings in eclampsia," British Journal of Obstetrics and Gynaecology, vol. 104, no. 10, pp. 1165-1172, 1997.

[11] T. Ergün, H. Lakadamyali, and A. Yilmaz, "Recurrent posterior reversible encephalopathy syndrome in a hypertensive patient with end-stage renal disease," Diagnostic and Interventional Radiology, vol. 14, no. 4, pp. 182-185, 2008.

[12] H. Kadikoy, W. Haque, V. Hoang, J. Maliakkal, J. Nisbet, and A. Abdellatif, "Posterior reversible encephalopathy syndrome in a patient with lupus nephritis," Saudi Journal of Kidney Diseases and Transplantation, vol. 23, no. 3, pp. 572-576, 2012. 
[13] O. H. Chang, A. Stanculescu, C. Dola, and W. B. Rothwell, "Recurrent posterior reversible encephalopathy syndrome potentially related to AIDS and end stage renal disease: a case report and review of the literature," Case Reports in Medicine, vol. 2012, Article ID 914035, 3 pages, 2012.

[14] M. A. Rizzo, F. Frediani, A. Granata, B. Ravasi, D. Cusi, and M. Gallieni, "Neurological complications of hemodialysis: state of the art," Journal of Nephrology, vol. 25, no. 2, pp. 170-182, 2012.

[15] H. Mu, H. Chai, P. H. Lin, Q. Yao, and C. Chen, "Current update on HIV-associated vascular disease and endothelial dysfunction," World Journal of Surgery, vol. 31, no. 4, pp. 632643, 2007.

[16] P. Gresele, E. Falcinelli, M. Sebastiano, and F. Baldelli, "Endothelial and platelet function alterations in HIV-infected patients," Thrombosis Research, vol. 129, no. 3, pp. 301-308, 2012.

[17] Y. C. Huang, P. L. Tsai, J. H. Yeh, and W. H. Chen, "Reversible posterior leukoencephalopathy syndrome caused by blood transfusion: a case report," Acta Neurologica Taiwanica, vol. 17, no. 4, pp. 258-262, 2008. 


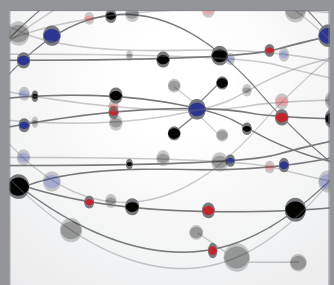

The Scientific World Journal
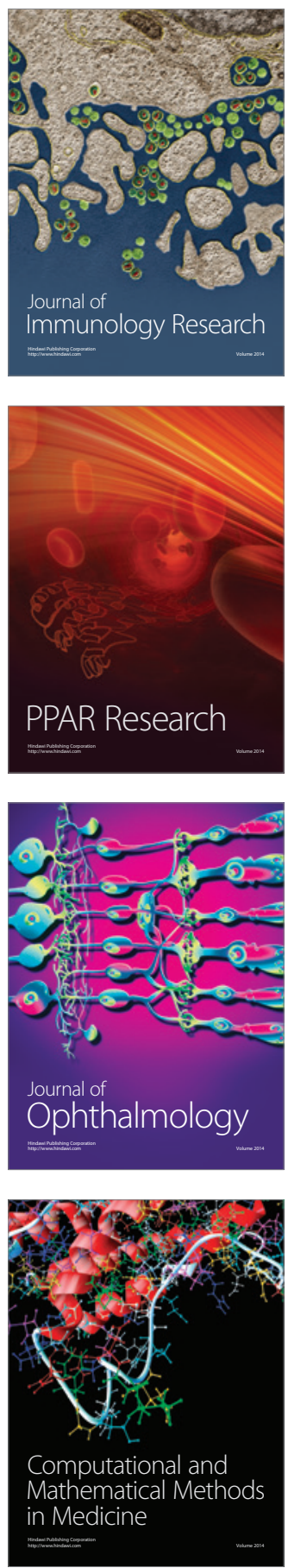

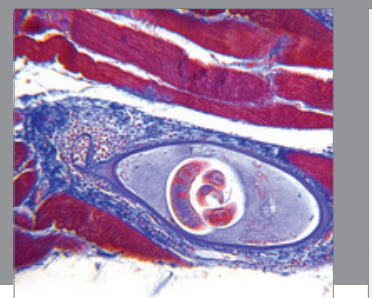

Gastroenterology

Research and Practice
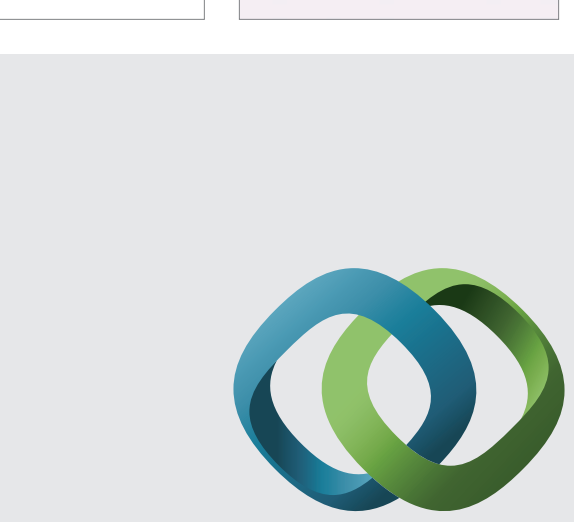

\section{Hindawi}

Submit your manuscripts at

http://www.hindawi.com
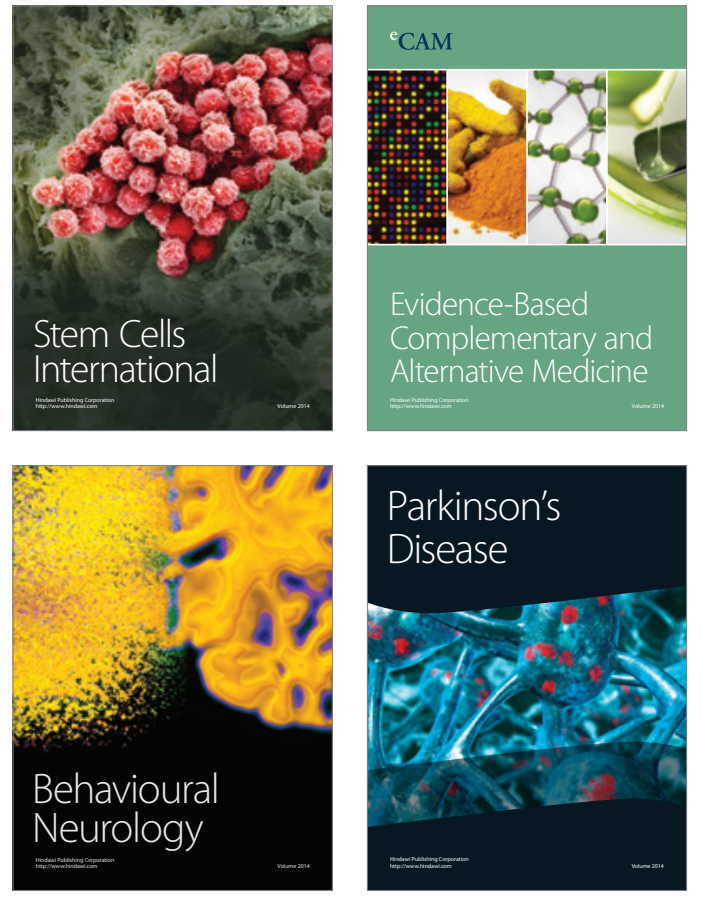
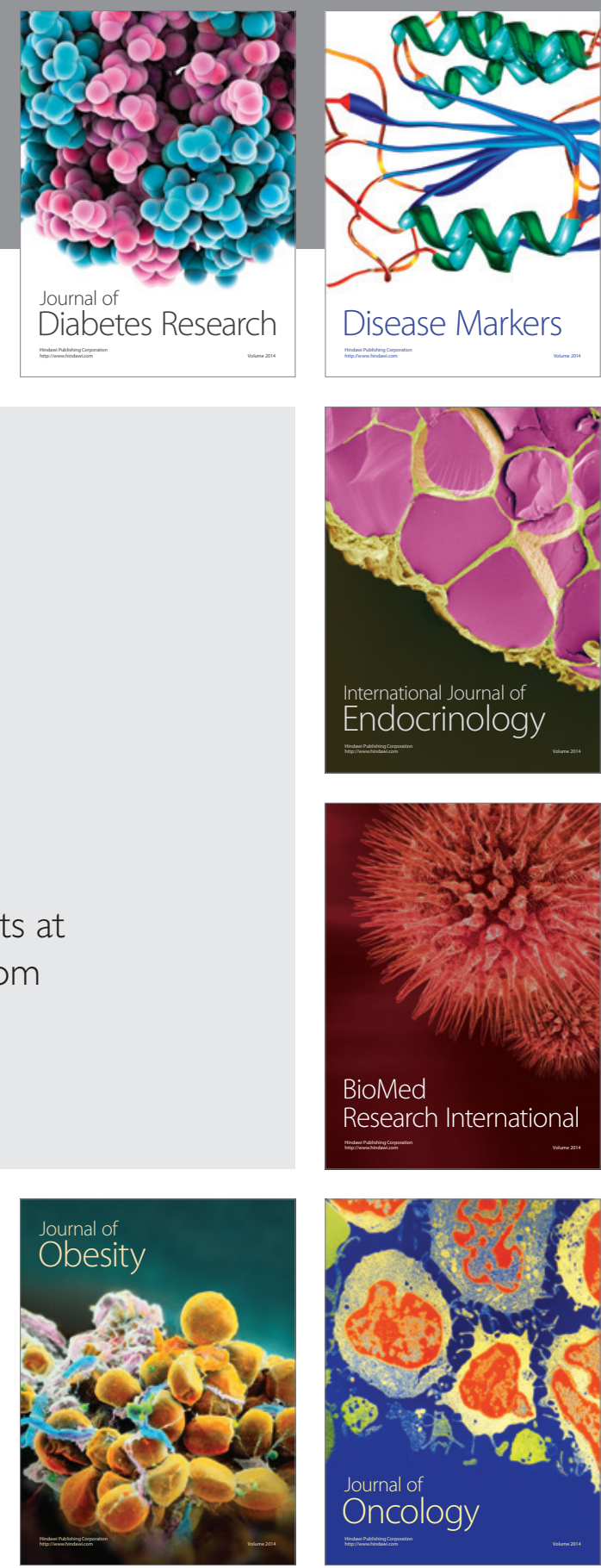

Disease Markers
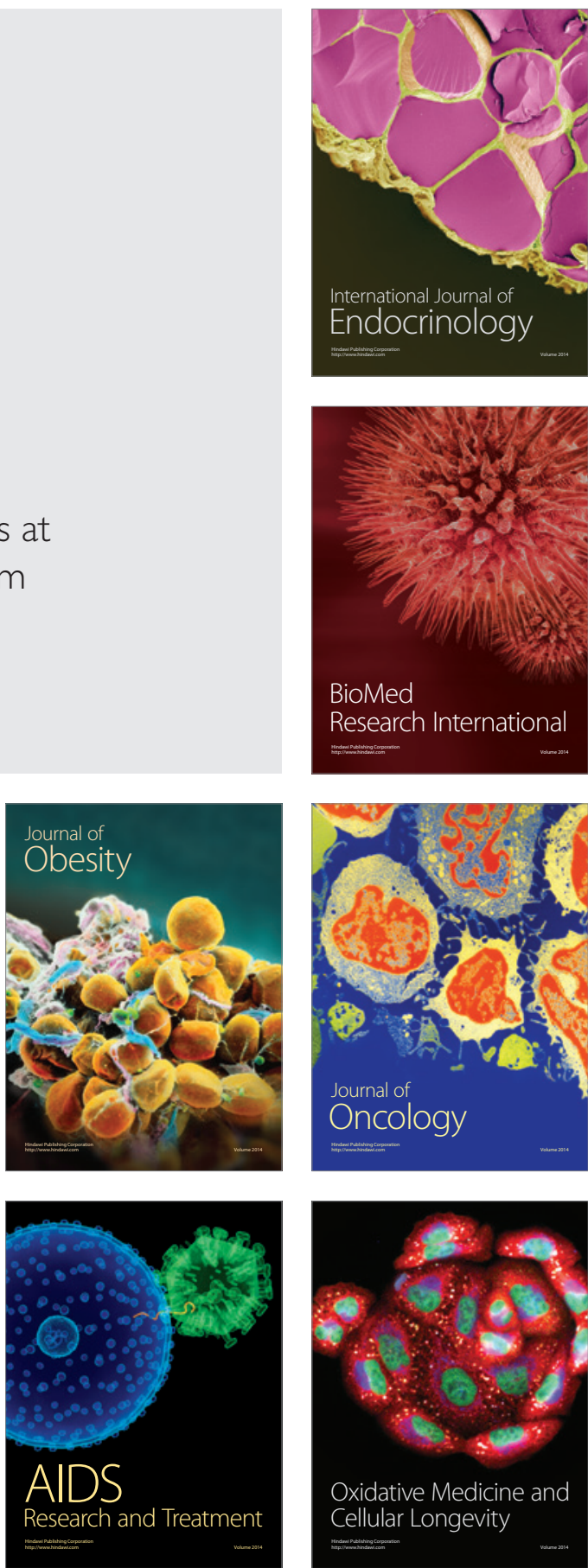